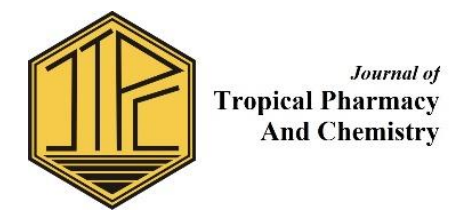

\title{
GUILLAIN-BARRE SYNDROME: A CASE REPORT
}

\author{
Nikunja Basini Pati, Ruksana Begum ${ }^{1, *}$, Soujanya. $\mathbf{R}^{1}$, Salome Satya Vani. $\mathbf{P}^{2}$ \\ ${ }^{1}$ Pharm. D \\ Pulla Reddy Institute of Pharmacy \\ Dommadugu(V), Gummadidala(M), SangaReddy (D), Telangana(S), India \\ ${ }^{2}$ Assistant Professor \\ Pulla Reddy Institute of Pharmacy \\ Dommadugu(V), Gummadidala(M), SangaReddy (D), Telangana(S), India \\ email: pssalom@gmail.com, ruksana1403@gmail.com, soujanya.r08@gmail.com
}

\begin{abstract}
Guillain-Barre syndrome (GBS) is a complicated degenerative neurological disorder which can be acute or chronic in nature. It is an acquired condition which is characterized by progressive, symmetrical, proximal and distal tingling and weakness. Muscle stretch reflexes are decreased to absent and loss of sensation is common. Etiology remains unclear but pathophysiology includes demyelination of spinal nerve roots. Death is rare. Early diagnosis and prompt referral should occur in severe cases due to the incidence of potential ventilation failure and cardiovascular instability in some patients. The case of a 26-year-old male presenting to a physician is described. The importance of a correct diagnosis by the physician and the subsequent management is reviewed.
\end{abstract}

Keywords: Guillain-Barre syndrome, extremity weakness, peripheral nerves, trauma

Submitted on: 02 January 2019

Accepted on: 03 June 2019

DOI: https://doi.org/10.25026/jtpc.v4i5.206

\section{INTRODUCTION}

Guillain-Barre Syndrome is a rare but serious autoimmune disease of the peripheral nervous system. GBS can affect people of any age or either sex, although it is slightly more common in older people and males. The condition usually begins following an infectious disease. The condition affects around 1 in 100,000 people in the United States (U.S.). In the case of GBS, the immune system attacks the myelin sheaths of peripheral nerves. As the myelin is damaged, nerves can no longer send certain information to the spinal cord and brain, such as touch sensations ${ }^{1}$. This causes the sensation of numbness. In addition, the brain and spinal cord are no longer able to transmit signals back to the body, leading to muscle weakness1. The disease often begins with tingling sensations and weakness in the feet and legs. It then slowly spreads 
upward until a large portion of the body is affected. The nerves connected to the lower extremities are the longest in the body. This travel distance makes these nerves more prone to a break in nerve signals due to GBS and its symptoms. Early diagnosis and prompt referral should occur in severe cases due to the incidence of potential ventilatory failure and cardiovascular instability in some patients. Nerve conduction exam, like Electromyography (EMG), lumbar puncture are the test used to diagnose the syndrome. Immunoglobulin therapy, plasma pheresis are the treatment options. The most common cause of acute muscle weakness associated with peripheral neuropathy in adults is Guillain-Barre syndrome $^{2}$

This paper will discuss the causes, history, incidence and pathology of Guillain-Barre syndrome. The case of 37year old male is also presented.

\section{CASE REPORT}

A 26 old male admitted in hospital with complaints of pedal edema, facial puffiness, shortness of breath (10 days), abdominal distension positive, fever, nausea, body pains. His symptoms began slowly following an upper respiratory tract infection that he had about 14 days prior. No history of trauma, diabetic mellitus, and hypertension was reported. And it's a known case of seizures on Eptoin 100mg.

Examination of this man revealed healthy person and looks in good condition. Generalized weakness of the lower extremities was noted when testing the quadriceps, hamstrings and the gastrocnemius-soleus muscle groups. A general physical examination gave no other orthopedic, neurological, or organic findings. Respiratory and cardiac functions shown shortness of breath and cardiomegaly simultaneously. The patient was referred to his physician for lab work. Blood tests were ordered and the findings included an increase in the white blood cell count. The conclusion drawn from this was that this patient had recently suffered from some type of infection which correlated well with his history of an upper respiratory tract infection.

Chest- X-ray shown cardiomegaly, creatinine phosphokinase test was normal. The patient was referred to his physician for lab work. Blood tests were ordered and the findings included an increase in the white blood cell count. The conclusion drawn from this was that this patient had recently suffered from some type of infection which correlated well with his history of an upper respiratory tract infection. Ultrasound of abdomen shown mild right pleural effusion, subcutaneous edema in bilateral legs more at the ankles noted. No evidence of dvt.

A diagnosis of Guillain-Barre syndrome was made after utilizing the criteria that strongly support the diagnosis of Guillain Barre Syndrome. Features required to rule out diagnoses other than Guillain Barre Syndrome would include: no history of hexacarbon abuse, no evidence of porphyria, no history or culture evidence of diptheria, no history or evidence of lead intoxication, symptoms not purely sensory, no evidence of poliomyelitis, botulism, toxic neuropathy, or tick paralysis ${ }^{2}$. Therapeutic management of this included rest, nutritional support, patient education and periodic assessment of motor, respiratory, and cardiac function. Chiropractic care, which included spinal manipulative therapy to areas of subjective complaint, was palliative. Medical management was done with dobutamine infusion to treat cardiac failure as his report shown cardiomegaly. Diuretic has given to treat edema. Antibiotics have given to treat infections. This patient slowly improved so that he could return to work. 


\section{DISCUSSION}

Guillain-Barre syndrome (GBS) is also known as poly radiculoneuritis, and chronic inflammatory demyelinating poly radiculopathy (CIDP) ${ }^{3}$. In North America it has an incidence of $1-7$ per 100,000 persons and as such is classified as one of the more common neurological conditions. It is the most common cause of acute weakness in patients under 40 years of age ${ }^{4}$.Diagnosis is difficult due to the lack of any test that is pathogenomonic of this condition. It is complicated disorder which can be chronic or acute in nature. It's etiology is unclear although it has been associated with both cell and humoral mediated autoimmune mechanisms ${ }^{5}$. Guillain-Barre syndrome represents an important acquired condition that is acutely evolving, is immune mediated, and an inflammatory disorder of the peripheral nervous system. It progresses leading to demyelination and axonal loss. Clinical hallmarks are symmetrical flaccid muscle paresis and areflexia in the presence of increased cerebrospinal fluid protein content and electro physiologic studies demonstrating evolving demyelination ${ }^{6}$. Causes such as viral infection ${ }^{7}$, surgery ${ }^{8,9}$, ${ }^{10}$, blood transfusion ${ }^{11}$, and myco plasma infection $^{12}$, vitamin B12 deficiency have been implicated as prodrome to this syndrome in many cases. The diagnosis of Guillain-Barre syndrome can be supported by the following clinical features as listed.

\section{Clinical features that strongly support the diagnosis of Guillain-Barre syndrome}

Upper respiratory tract infection

Progression over days to weeks

Mild sensory signs or symptoms

Onset of recovery after 2 to 4 weeks of progression

Elevated cerebrospinal fluid protein after 1 week of symptoms
Abnormal results of electro diagnostic studies, with slowed conduction or prolonged $\mathrm{F}$ waves

Autonomic dysfunction

Most patients develop weakness which tends to begin in the lower extremities due to demyelination of the peripheral nerves resulting in ascending paralysis and also loss of cranial nerve function $^{12}$. Manifestations may be acute or chronic, and temporary or permanent, depending upon the degree of neuronal destruction ${ }^{13}$. Muscle stretch reflexes are depressed in most patients and the sensory loss is variable. Difficulty with walking, running, climbing stairs, and getting up from chair are usual early complaints. This weakness is usually symmetric and can also involve the upper extremities. ${ }^{14}$

Of importance is the gamut of conditions that should be differentiated. Hexa carbon abuse, as in glue sniffing, can simulate Guillain-Barre syndrome. Diptheria presents similar findings in its later stages; therefore recent infection of diphtheria must be taken into consideration $^{15}$. Others that should be considered are poliomyelitis, any evidence of porphyria, botulism, lead or arsenic intoxication, tick paralysis as in Lyme disease, and acute toxic neuropathies (from organ phosphorus compounds). It is therefore important to accurately and thoroughly evaluate any cause of acute weakness ${ }^{16}$.

An objective index for diagnosis can be made utilizing motor evoked potentials (MEP). Cui found in 27 patients with GBS that the findings of MEP were consistent with pathological changes of demyelination and clinical distribution of muscle weakness. good correlation between abnormal MEP and degree of muscle weakness (p 0.01) was noted. He also found that MEP can be used for differential diagnosis as well as follow-up and effect of therapy ${ }^{17}$. MEP is painless and can be done easily and repeated. The 
pathogenesis of Guillain-Barre syndrome shows edematous changes proximal to the spinal nerve root at the junction of the anterior and posterior roots. The myelin sheaths soon become irregular at about the third day. Lymphocytes appear about the ninth day and phagocytosis on the eleventh day. These findings were found in severe cases of this syndrome that led to death ${ }^{18}$.

Researchers believe that the myelin destruction is limited to those areas of nerve trunks with intense inflammation suggesting that the inflammatory cells have direct effect initiating the demyelination. The demyelination occurs primarily in areas infiltrated with inflammatory cells. Degeneration of the basement membrane of the Schwann cell results complication ${ }^{19}$. This is due to macrophages in the presence of lymphocytes and not lymphocyte by themselves ${ }^{20}$.This breakdown, which is unclear, is associated with an autoimmune attack on component of peripheral myelin $^{5,21}$. This attack is also mediated by $\mathrm{T}$ cells ${ }^{21}$.

\section{Treatment:}

Treatment for patients with Guillain-Barre syndrome depends on whether they have mildly acute, severely acute or chronic involvement. The incidence of death in one study was $1.5 \%$ to $8 \%$ of patients ${ }^{22}$. Another study listed the death rate at $4 \%{ }^{23}$.Other common complications include ventilatory failure and cardiovascular instability for which intensive care support should be utilized. Ventilatory failure is caused by involvement of airway and respiratory muscles, particularly the diaphragm. Cardiovascular instability is due to involvement of the autonomic nervous system and results in labile blood pressure, cardiac arrhythmias, and hypovolemia. Compressive neuropathies occur in patients with protracted weakness and are an important cause of residual neurological deficits. The use of corticosteroids demonstrated no benefit ${ }^{23}$. The only well-investigated efficacious immune modulator therapy is plasmapheresis $^{24}$. Plasma pheresis has been shown to decrease ventilator dependence in severe Guillain-Barre syndrome. No irreversible complications of plasmapheresis were observed. In all cases of this condition special emphasis should be given to psychological support and management of pain $^{24}$.persistent residual paresis occurs in severe cases and high majority of these patients ultimately have good functional recovery and can recover completely at about 12 months after onset ${ }^{23}$.

\section{CONCLUSION}

Guillain-Barre syndrome is neurological disorder resulting primarily in muscle paralysis that in most cases is symmetrical. Patients may have mild involvement or severe involvement which may in small percentage lead to death. Patients may present to chiropractic office with symptoms of polyradiculoneuralgia which may at first be interpreted as radicular pain pattern of spinal origin. It is extremely important to identify and urgently refer; potential severe cases in order to have the appropriate investigations (i.e. electrodiagnostic studies, spinal tap) instituted and have the appropriate care administered. Differential diagnosis is of utmost importance.

\section{REFERENCES}

[1] Guillain-Barré syndrome: How could it affect me? Last updated Tue 19 December 2017 By Tim Newman Reviewed by Stacy Sampson, DO

[2] Miller RG. Guillain Barre syndrome. Postgraduate Medicine 1985; 77(7):57-64.

[3] Mendell JR. Chronic inflammatory demyelinatin 
polyradiculopathy. Annual Review Medicine 1993; 44:211219.

[4] Kraft GH, Freal MS, Coryell JK. Disability, disease duration and rehabilitation service needs in multiple sclerosis: patient perspectives. Arch Phys Med Rehab, 1986; 67:164.

[5] Murray DP. Impaired mobility: Gullain-Barre syndrome. J Neuroscience Nursing 1993; 25(2):100-104.

[6] Hund EF, Borel CO, Comblath DR, Hanley DF, McKhann GM. Intensive management and treatment of severe Guillain-Barre syndrome. Critical Care Medicine 1993; 21(3):433-446.

[7] Ormerod IE, Ceekerell OC. Guillain-Barre syndrome after herpes zoster infection, a report of 2 cases. European Neurology 1993; 33(2):136-318.

[8] Stambough JL, Quinlan JG, Swanson JD. Guillain-Barre syndrome following spinal fusion for adult scoliosis. Spine 1990;15(1):45-46.

[9] Ennis JH, Bednar DA. Lumbosacral fusion in a patient with recurring Guillain-Barre syndrome and acute brachial neuritis. J Spine Disord 1992; 5(2):217-218.

[10] Ginn D. Guillain-Barre syndrome. An uncommon but severe illness. Postgrad Med 1991; 90(5):145416, 153-156.

[11] Merelli E, Sola P, LaSpina I, Orlando A, Milanti G. GuillainBarre polyradiculoneuritis after blood transfusion. Ital J Neuro Sci 1991; 12(3):313-315.

[12] Nadkarni N, Lisak RP. GuillainBarre syndrome (GBS) with bilateral optic neuritis and central white matter disease. Neurology
1993; 43(4):842-843.

[13] Murray DP. Impaired mobility: Gullain-Barre syndrome. J Neuroscience Nursing 1993; 25(2):100-104.

[14] Mendell JR. Chronic inflammatory demyelinating polyradiculopathy. Annual Review Medicine 1993; 44:211219.

[15] Macleod J. Davidson's Principles and Practice of Medicine. Eleventh Edition, New York: Churchill Livingstone 1975:6568.

[16] Felt H, Tindall RS, Glasberg M. Sources of error in the diagnosis of Guillain-Barre syndrome. Muscle Nerve 1982; 5(2): 111117.

[17] Cui LY. Magnetic stimulation motor evoked potential in patients with Guillain-Barre syndrome (Chinese). Chung-Mua I Msueh Tsa Chih 1992; 72(10);600-603, 639.

[18] Haymaker W, Kemohan JW. Landry-Guillain-Barre syndrome: clinicopathologic report of 50 fatal cases and a critique of the literature. Medicine (Baltimore) 1949; 28:59-141.

[19] Murray DP. Impaired mobility: Gullain-Barre syndrome. J Neuroscience Nursing 1993; 25(2):100-104.

[20] Prineas JW. Pathology of the Guillain-Barre syndrome. Ann Neurol 1981; 9(suppl):6-19.

[21] Hartung HP. Immune-mediated demyelination (comment). Annals Neurology 1993; 33(6):583-587.

[22] Ropper AH. Management of Guillain-Barre syndrome. In: Ropper AH, Kennedy SK, Zervos NT, Ed. Neurological and neurosurgical intensive care. Baltimore: University Park 
Press, 1983:163.

[23] Smith GD, Hughes RA. Plasma exchange treatment and prognosis of Guillain-Barre syndrome. Quarterly J Medicine 1992; 85(306):751-760.
[24] Hund EF, Borel CO, Comblath DR, Hanley DF, McKhann GM. Intensive management and treatment of severe Guillain-Barre syndrome. Critical Care Medicine 1993; 21(3):433-446. 